\title{
Neuroendocrine, Psychophysiological and Subjective Reactivity to an Alcohol Placebo in Male Alcoholic Patients
}

\author{
Zelig S. Dolinsky, PhD, David E. Morse, PhD, Richard F. Kaplan, PhD, Roger E. Meyer, MD, Douglas Corry, and
} Ovide F. Pomerleau, PhD

\begin{abstract}
Alteration in neuroendocrine activity associated with the regulation of energy metabolism and food intake may play a role in characterizing the alcohol dependent state. Alcoholics, when compared to controls, demonstrated significantly larger and more rapid glucose and insulin responses following the consumption of a placebo beer, which they believed contained alcohol. The existence of significant correlations between peak neuroendocrine responses and desire to drink, anxiety, as well as psychophysiological responses in alcoholics suggests the potential multivariate nature of the biological/ behavioral state associated with alcohol dependence.
\end{abstract}

A HALLMARK of alcohol dependence is "craving," a strong subjective desire to drink. Craving is usually not apparent in treatment settings where alcohol is not available to the alcoholic. ${ }^{\prime}$. These clinical observations suggest that stimuli, associated with availability of, or with actual alcohol consumption, are necessary to elicit craving.

We have previously reported that, in contrast to nonalcoholic subjects, alcoholics experienced autonomic arousal in association with increased reported desire to drink, following the presentation of alcohol-related stimuli. $^{2,3}$ In addition, the increased desire to drink among alcoholic subjects was significantly related to their belief that they were drinking real beer, regardless of whether they received real beer or placebo. ${ }^{2}$ We have extended these observations by demonstrating that alcoholics experienced significantly more craving and salivated more than nonalcoholic controls when exposed to an open bottle of their favorite beverage. ${ }^{4}$ Furthermore, salivary response was highly correlated with positive expectations concerning the behavioral and cognitive effects of drinking. ${ }^{5}$

The finding of increased salivation in alcoholics takes on added significance given the growing interest in com-

From the Alcohol Research Center, Department of Psychiatry, University of Connecticut Health Center, Farmington, Connecticut, and, Department of Psychiatry, University of Michigan, Ann Arbor, Michigan.

Received for publication February 14, 1986; revised manuscript received September 10, 1986; accepted September 18, 1986.

This research was supported in part by National Institute on Alcohol Abuse and Alcoholism Grants 5 P50 AA03510-08 and 5 T32 AA0729006.

Reprint requests: Zelig S. Dolinsky, PhD, Department of Psychiatry, University of Connecticut Health Center, Farmington, CT 06032-9984.

Copyright (C) 1987 by The American Medical Society on Alcoholism and The Research Society on Alcoholism. monalities underlying addictive and consummatory disorders and reports linking brain-gut neuropeptides to alterations in ethanol intake. ${ }^{6}$ Rodin $^{7}$ demonstrated that insulin response was exaggerated in externally responsive overweight individuals presented with food-related stimuli, and that hyperinsulinemia, independent of blood glucose level, was directly related to variables associated with increased hunger. ${ }^{8}$ Animal studies have shown that glucose intolerance increases ethanol intake, ${ }^{9}$ while subcutaneous insulin injection has been reported to both increase ${ }^{10}$ and decrease ${ }^{11}$ ethanol intake. At the clinical level, it has been demonstrated that length of sobriety was increased in alcoholics who chose diets containing twice as much sugar added to beverages and who exhibited greater overall carbohydrate intake. ${ }^{12}$

Research findings contrasting insulin and glucose responses in alcoholics and controls, before or after alcohol consumption, remain contradictory, possibly due to factors such as alcohol dosage, concurrent liver disease, and nutritional status. ${ }^{13}$ The interrelationships among altered insulin, glucose response and craving, as well as other subjective and psychophysiological states associated with alcohol consumption, are not well understood.

The present study is part of a continuing effort to define the biological, psychophysiological, and subjective state associated with alcohol dependence. The study specifically compared changes in plasma glucose, insulin, glucagon, and cortisol to measures of psychophysiology as well as anxiety and desire to drink following olfactory and visual stimulation by real beer and the consumption of placebo beer, contrasting the responses of alcoholic and control subjects in a repeated-measures design.

\section{METHODS}

\section{Subjects}

Eight male, alcoholic inpatients (DSM-III Alcohol Dependence diagnosis) were recruited from the ADATC (Alcohol and Drug Abuse Treatment Center), University of Connecticut Health Center and participated in this study during their second week of a 3-week treatment program. Nine control subjects (moderate drinkers) were recruited from the local community. One alcoholic subject did not rate beer as his beverage of choice, however, he did drink beer on occasion. All other control and alcoholic subjects rated beer as their first or second beverage of choice. Mean weight and age ( $\pm \mathrm{SE}$ ) for the alcoholic and control samples were: $174.2 \pm 11.8 \mathrm{lbs}$ vs. $173.7 \pm 7.6 \mathrm{lbs}(\mathrm{NS})$; and $32 \pm 2.3$ years vs. $24 \pm$ 
1.4 years $(p<0.025)$, respectively. All subjects were free of any major medical and psychiatric disorders and were not currently using any prescription or nonprescription drugs which might alter endocrine status. Liver enzyme values (GGTP, SGOT, SGTP) were recorded for the alcoholic inpatients on the day of admission to the ADATC. A 2-hr postprandial glucose determination (obtained 2-hr after lunch) was performed on all subjects prior to experimental participation. In the month before the study, controls consumed an average of $13.9 \pm \mathrm{SE} 4.1 \mathrm{oz}$ of absolute ethanol and alcoholics consumed $195.2 \pm 33.8 \mathrm{oz}$ of absolute ethanol in the month prior to their admission to the treatment unit. All alcoholic subjects were abstinent for at least 2 weeks prior to participation in the study. Controls did not consume any alcoholic beverages for at least $12 \mathrm{hr}$ before the study. Subjects were informed that they may or may not receive ethanol. Written informed consent was obtained after the nature and possible consequences of the study had been fully explained. Subjects were paid $\$ 30$ for participating in the study.

\section{Procedure}

On the day of the study, subjects chose a lunch from a number of low carbohydrate foods and beverages. Subjects were instructed to fast from 12:30 until the beginning of the lab session at 4:30 PM. At this time, subjects were seated in a comfortable chair and electrodes for measuring heart rate, skin conductance level and earlobe temperature were connected. Psychophysiological response as well as subjective measures of anxiety and desire to drink were recorded. Heart rate was determined by a Cardio-Tach recorder (model CT46001302) from an infrared finger sensor. Electrodermal changes (skin conductance level) were recorded on an Autogen 3400 dermagraph using silver chloride electrodes. Earlobe temperature was monitored on an Autogen 1000B feedback thermometer. These analogue signals were digitized on line by a $128 \mathrm{k}$ word MF211 microcomputer and saved on floppy disks for later data analysis. Anxiety and desire to drink were measured on a 1 to 5 scale with 1 indicating "No," and 5 indicating "Greatest" anxiety or desire to drink.

Following these measures, an $18 \mathrm{G}$ catheter needle (connected to a 20 inch intravenous line which passed through a one-way mirror in the wall of the testing chamber) was inserted into a vein in the antecubital region of the left arm and the arm was draped. A preliminary blood sample was then drawn (1st baseline: B1). Following a 45-min adaptation period (during which subjects filled out questionnaires assessing drinking history) desire to drink and psychophysiological responses were assessed again and a blood sample was drawn (2nd baseline: B2). All subjects were then informed that they would be presented with a real beer. They were told not to drink the beverage until told to do so in a few minutes. Subjects then were instructed to hold, smell and think about, for a 3min period, a real beer (Pabst Blue Ribbon) which was presented in a frosted mug. Following this presentation, a breathlyzer test was performed. During the testing the real beer was removed and replaced with a placebo beer (Steinbrau, malt beverage, Eastern Brewing Corporation, Hammonton, NJ) without the subject's knowledge. Subjects were then instructed to drink the beer (actually placebo) within a 5-min period. Measures of anxiety, desire to drink, psychophysiology, and blood samples were obtained during the real beer presentation and placebo consumption (Fig. 1).

All whole blood samples were immediately placed on ice prior to centrifugation. Blood samples for glucose analysis were preserved with $20 \mathrm{mg}$ of sodium fluoride and $2 \mathrm{mg}$ of thymol. Blood samples were spun for $10 \mathrm{~min}$ at $1500 \times \mathrm{g}$ and plasma aliquots were stored at $-70^{\circ} \mathrm{C}$ prior to assay. Plasma insulin, glucagon, and cortisol levels were determined by standard RIA procedures using kits employing the double antibody method: Insulin, Serono kit cat. \#2210000; Glucagon, Radioassay Systems Laboratory kit cat. \#133; Cortisol, Travenol kit cat. \#CA-529. Plasma glucose levels were determined on a Chemetrics Auto Analyzer using a glucose reagent (GDH-endpoint) supplied by Seragen, cat. \#45942.

\section{Data Analyses}

Neuroendocrine and psychophysiology data were analyzed utilizing a two-factor repeated measures ANOVA with groups Control and Alcoholic as the between-subjects factor and time as the repeated measure. Two ANOVA's were performed. In the first, the dependent measures were examined at the two baseline (B1, B2) time periods. This analysis was done to assess differences between groups with respect to adaptation following catheter insertion. The second ANOVA utilized data spanning from $B 2$ to the final time point in the study (postdrink, $61 \mathrm{~min}$ ) and was performed to determine differences between groups relative to the second baseline period. Subjective measures of desire to drink and anxiety were also analyzed using a repeated measures ANOVA with group as the between-subjects factor and time: B2 through postdrink, $61 \mathrm{~min}$ (desire to drink); B1 to postdrink, $4 \mathrm{~min}$ (anxiety), as the within-subjects measure, respectively.

In addition, relationships between neuroendocrine variables and other measures (anxiety, desire to drink, psychophysiology) were examined by calculating univariate correlations (Pearson's $r$, one-tailed) between neuroendocrine variables (peak response as percentage of $\mathrm{B} 2$ levels) and anxiety, desire to drink, as well as psychophysiological responses. Where appropriate the later measures (anxiety, desire to drink, psychophysiology) were expressed as percent of B2 to account for individual differences in baseline measures. Correlations between neuroendocrine responses, subjective report, and psychophysiological variables were examined separately in controls and alcoholics to assess potential group differences in the association among these variables.

\section{RESULTS}

\section{Neuroendocrine Responses}

With the exception of cortisol, alcoholic and control subjects displayed similar hormone levels at baseline (B1 and B2) and demonstrated similar significant decreases in plasma hormone concentrations between B1 and B2 (Fig. 2) (time: glucose, $F(1,15)=11.9, p<0.01$; insulin, $F(1,15)$ $=9.15, p<0.01$; glucagon, $F(1,15)=6.5, p<0.05)$. Cortisol values were depressed in alcoholics at B2 and remained so throughout the study (group $F(9,135)=7.51$, $p<0.05$ ). Alcoholics, when compared to controls, demonstrated significantly larger and more rapid glucose and insulin responses (Fig. 2) following the consumption of the placebo beer, which they believed contained alcohol (group $\times$ time: glucose, $F(9,135)=2.28, p<0.05$; Insulin, $F(9,135)=3.5, p<0.001$. Glucagon responses following baseline were unremarkable and were similar in alcoholics and controls.

Alcoholics and controls did not differ in glucose, insulin, or glucagon concentrations during the beer presentation (predrink) phase of the study (note that although alcoholics demonstrated lower cortisol levels than controls, this effect was already observed at B2). Alcoholics and controls did not differ with respect to $2-\mathrm{hr}$ postprandial glucose levels which were assessed before the laboratory session (Mean $\pm \mathrm{SE}, 95 \pm 4.4$ vs. $89 \pm 2.1$, respectively).

\section{Subjective Report Data}

Both alcoholics and controls displayed an increase in desire to drink during the presentation phase of the study (time: $F(3,45)=5.14, p<0.05$, Fig. 3). Alcoholics tended 
TIME LINE FOR MEASURES
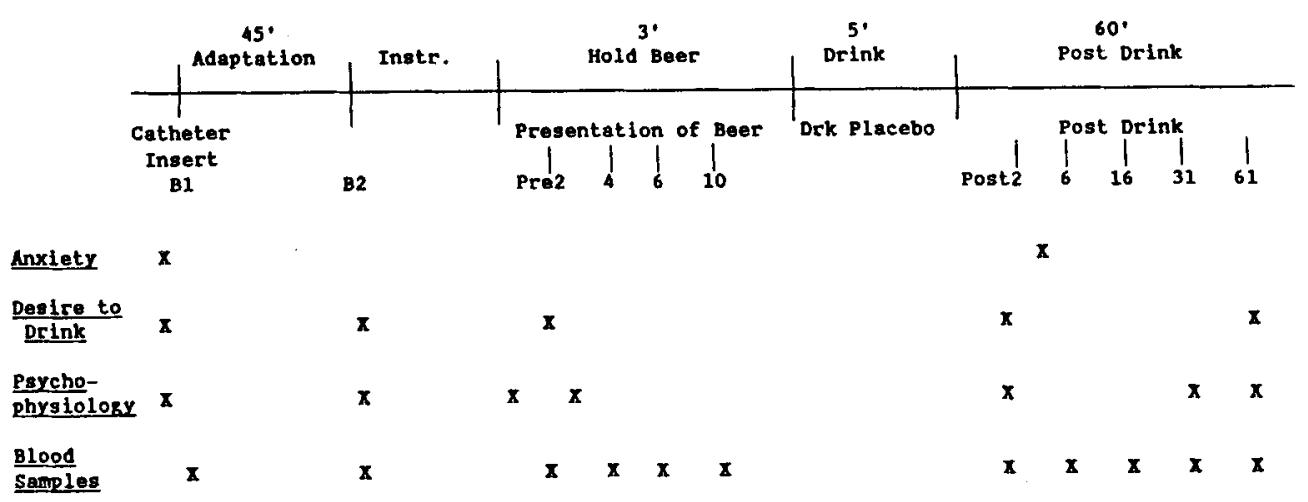

Fig. 1. Time course of study in minutes. $X$ 's mark the time at which a given subjective measure, psychophysiological response, or blood sample was obtained.

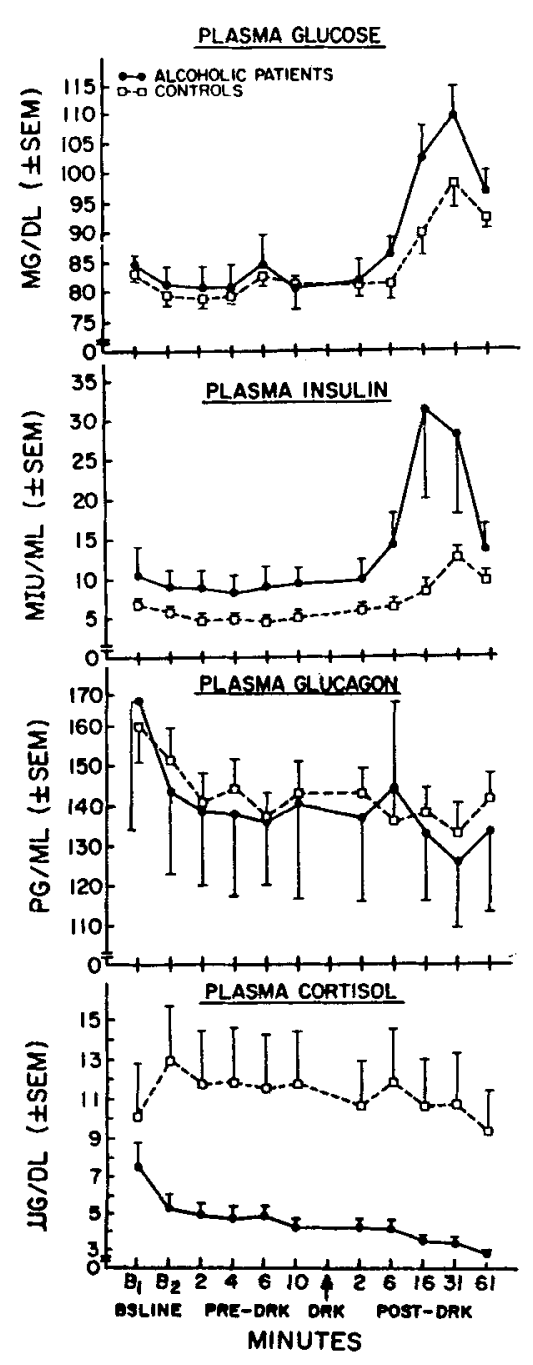

Fig. 2. Neuroendocrine levels (raw vaiues) in control and aicoholic subjects Changes from $\mathrm{B} 1$ to $\mathrm{B} 2$ were assessed utilizing a two-way repeated measures ANOVA with group (control vs. alcoholic) as the between subjects measure and time (B1 to B2) as the within subjects measure.

Neuroendocrine results from the Predrink and Postdrink periods were evaluated relative to $\mathrm{B} 2$ utilizing a similar repeated measures ANOVA as used in the B1-B2 analyses; however, time was extended from $B 2$ through Postdrink 61 min.

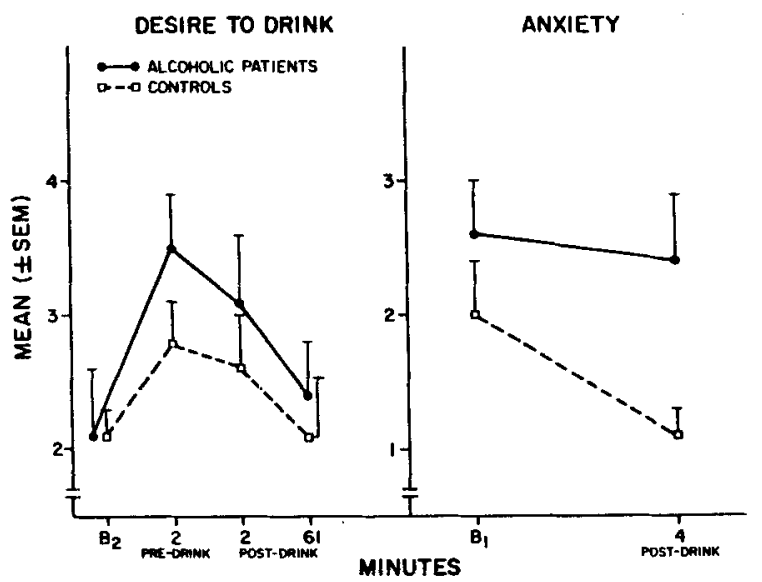

Fig. 3. Subjective measures of desire to drink and anxiety were analyzed utilizing a repeated measures ANOVA with Grp as the between-subjects factor and Time as the within-subjects measure.

to be more anxious than controls during the two periods when anxiety was measured (group: $F(1,15)=3.74, p=$ 0.072 , Fig. 3).

\section{Psychophysiological Data}

Alcoholics showed greater mean heart rates at $\mathrm{B} 1$ and B2 relative to controls (B1: 76 vs. 65 ; B2: 71 vs 65 , Group: $F(1,14)=5.45, p<0.05)$. The alcoholic group tended toward an increase in heart rate during the presentation and immediately following consumption; however, this effect did not reach statistical significance. Both groups showed increases in skin conductance from B1 to B2 (alcoholics: 13.6 to 20.9 micromhos; controls: 11.5 to 18 $\mu$ mhos, time: $F(1,14)=25.2, p<0.01)$. Skin conductance levels in both groups tended to show a small increase following the initial beverage presentation and then a gradual decrease throughout the remainder of the study; however, none of these effects reached statistical significance. Earlobe temperature was greater in the alcoholics at both baseline periods (alcoholics: $95.4,95.1^{\circ} \mathrm{F}$ vs. controls: 92.6, 91.8; Group $F(1,14)=5.53, p<0.05$ ). (Note: only $14 d f$ are reported for the psychophysiology 
data because one control subject's data were missing due to technical difficulties associated with the data collection). In addition, both controls and alcoholics showed a decrease in earlobe temperature following beverage consumption, with alcoholics tending to show a greater decrease: $3^{\circ} \mathrm{F}$ vs. $1^{\circ} \mathrm{F}$ (group $\times$ time: $F(5,70)=2.13, p=$ 0.07).

\section{Intercorrelation Results}

As shown in Table 1, alcoholics displayed significant relationships between glucose response (at $31 \mathrm{~min}$ postdrink, i.e., peak response) and baseline as well as postdrink heart rate. In addition, insulin response (at 16 min postdrink, i.e., peak response) was significantly related to baseline desire to drink, presentation and postdrink skin conductance level and baseline earlobe temperature. Furthermore, glucagon response (at $6 \mathrm{~min}$ postdrink, i.e., peak response) was correlated with both postdrink anxiety and heart rate and both presentation and postdrink earlobe temperature. It should be noted that both glucose and insulin postdrink peak responses were most highly correlated with desire to drink and psychophysiological responses which occurred prior to or only shortly after beverage consumption. This pattern was not as evident for the glucagon response relationships.

There were no significant correlations among neuroendocrine peak responses and either desire to drink or anx-

\begin{tabular}{|c|c|c|c|c|c|}
\hline Alcoholics & Glucose & Insulin & Glucagon & $\begin{array}{c}\text { Pre- } \\
\text { anxiety }\end{array}$ & $\begin{array}{l}\text { Post- } \\
\text { anxiety }\end{array}$ \\
\hline \multicolumn{6}{|l|}{ Anxiety } \\
\hline Predrink & 0.33 & 0.58 & -0.18 & & -0.37 \\
\hline Postdrink & -0.27 & 0.32 & $0.82^{*}$ & & \\
\hline \multicolumn{6}{|l|}{ Desire to drink } \\
\hline 2nd baseline & 0.43 & $0.72^{\star}$ & 0.37 & 0.12 & 0.43 \\
\hline Presentation & 0.03 & -0.35 & -0.09 & -0.11 & 0.06 \\
\hline Postdrink 2 min & -0.48 & -0.46 & 0.40 & -0.32 & 0.38 \\
\hline Postdrink $60 \mathrm{~min}$ & -0.54 & -0.28 & 0.37 & -0.30 & 0.54 \\
\hline \multicolumn{6}{|l|}{ Heart rate } \\
\hline 2nd Baseline & $0.83^{\star}$ & 0.53 & -0.28 & 0.16 & 0.09 \\
\hline Presentation & -0.29 & 0.18 & 0.50 & 0.53 & 0.00 \\
\hline Postdrink 2 min & $-0.66 t$ & -0.22 & $0.62 \dagger$ & -0.14 & 0.51 \\
\hline Postdrink $60 \mathrm{~min}$ & -0.34 & 0.11 & 0.08 & -0.33 & 0.47 \\
\hline \multicolumn{6}{|c|}{ Skin conductance level (SCL) } \\
\hline 2nd Baseline & -0.02 & -0.51 & -0.31 & -0.36 & -0.45 \\
\hline Presentation & 0.31 & $0.63 \uparrow$ & -0.11 & 0.54 & 0.01 \\
\hline Postdrink 2 min & 0.12 & $0.79^{*}$ & 0.13 & $0.68 t$ & 0.06 \\
\hline Postdrink 60 min & 0.31 & -0.37 & -0.12 & -0.25 & -0.08 \\
\hline \multicolumn{6}{|l|}{ Temperature } \\
\hline 2nd Baseline & 0.29 & $0.71^{*}$ & -0.24 & 0.37 & 0.03 \\
\hline Presentation & 0.38 & 0.11 & $-0.63 \dagger$ & 0.35 & -0.49 \\
\hline Postdrink 2 min & 0.00 & 0.00 & -0.16 & -0.17 & -0.15 \\
\hline Postdrink $60 \mathrm{~min}$ & 0.58 & 0.26 & $-0.66 t$ & 0.22 & -0.59 \\
\hline
\end{tabular}

$p<0.01$.

$\dagger p<0.05$.

For these correlations all neuroendocrine responses are based on percent of the level at second baseline (B2), and are measured at the peak of response in the alcoholic group for a given measure (i.e., Insulin, Postdrink 16; Glucose, Postdrink 31; Glucagon, Postdrink 6 min). Likewise, to take into account baseline effects for the psychophysiology and subjective report measures, correlations are based on percent of baseline response for those correlations involving time periods foilowing the initial measurement. (Note: to limit the number of correlations, psychophysiology data are only reported for the time points at which desire to drink was collected.) iety in the control group. Interestingly, only baseline heart rate correlated with peak glucose response (at $31 \mathrm{~min}$ postdrink; $r=-0.72, p<0.01$ ) in the control group and this correlation was opposite to that observed in the alcoholic group $(r=0.83, p<0.01)$.

\section{Background Variables}

Although controls were significantly younger than alcoholics, age was unrelated to peak neuroendocrine responses in either group (neuroendocrine responses expressed as per cent of B2). Body weight and 2-hr postprandial glucose levels were unrelated to neuroendocrine responses in the alcoholics, although these variables were significantly related to insulin in the controls $(r=0.59, p$ $<0.05$ ). Mean ( \pm SE) liver enzyme values for the alcoholic group were: GGTP, $80.5 \pm 31.9$; SGOT, $44.6 \pm 13.5$; SGPT, $64.8 \pm 27.8$. In the alcoholic sample, peak neuroendocrine responses were unrelated to either admission liver enzyme values or to level of alcohol consumption as measured by the amount of absolute ethanol consumed in the month before admission. The amount of malt beverage consumed contained less than $1 \mathrm{~g}$ of glucose. Previous work utilizing healthy volunteers has demonstrated that approximately $25 \mathrm{~g}$ of glucose ingested orally results in approximately a $10 \mathrm{mg} / 100 \mathrm{ml}$ increase in plasma glucose during a 30 -min measurement period. ${ }^{14}$ Therefore, the glucose contribution of the nonalcoholic beverage did not seem sufficient to account for the magnitude of the glucose or insulin response in either the control or alcoholic samples.

\section{DISCUSSION}

The present paper represents a multidimensional approach to examining the alcohol dependent state. Our findings include the demonstration of a shorter latency and increased magnitude of both glucose and insulin responses in alcoholic subjects following the consumption of a placebo beverage. The existence of significant correlations between the peak neuroendocrine responses and desire to drink, anxiety, as well as psychophysiological responses in alcoholics suggests the potential multivariate nature of the biological/behavioral state associated with alcohol dependence. We believe that future research should focus on an understanding of the roles of these interacting variables in mediating abnormal alcohol consumption. As a first attempt at combining information across a number of physiological systems we are aware of the limitations of a correlational approach, particularly with respect to the likelihood of spurious correlations. Therefore, current work in our laboratory is directed at replicating and extending our present findings.

The possibility that the observed hormonal responses may be simply due to the inability of the liver to metabolize glucose or insulin seems unlikely, since controls and alcoholics exhibited similar 2-hr postprandial glucose lev- 
els as well as similar glucose, insulin, and glucagon levels at baseline. Additionally, level of alcohol consumption in the month prior to hospital admission and liver enzyme values at admission were unrelated to hormonal responses in the alcoholics. However, we acknowledge the possibility that our neuroendocrine findings may be secondary to the physiological changes associated with heavy alcohol consumption rather than related to the mechanisms involved in the addiction process.

The finding of an equivalent decrease in glucose and insulin levels from the first to the second baseline in controls and alcoholics suggests that the two groups do not differ with respect to glucose and insulin responses to an acute nonspecific stressor (catheter insertion). It is interesting to note that cortisol may not be a reliable indicator of anxiety or stress in alcoholics since this hormone was depressed throughout the study. It has been reported that alcoholics demonstrate a reduced cortisol response to various stressors, suggesting an alteration in the sensitivity of the hypothalamic-pituitary-adrenal axis. ${ }^{15}$ Alternatively, decreases in cortisol may be associated with a compensatory response in the alcoholic sample since chronic ethanol ingestion in alcoholic patients is reported to increase cortisol levels. ${ }^{16}$

In examining the results of this study it appears as if the changes in neuroendocrine responses observed in the alcoholic subjects following the consumption of a placebo beer are associated with psychophysiological and subjective responses generally occurring prior to or shortly following beverage consumption. Future research in our laboratory will be more closely directed at addressing the time course of interrelationships of changes in these variables with respect to the roles they play in mediating the alcohol dependent state.

\section{REFERENCES}

1. Cooney NL, Baker LH, Pomerleau OF: Cue exposure for relapse prevention in alcohol treatment, in Craig KD, McMahon RJ (eds):
Advances in Clinical Behavior Therapy. New York, Brunner/Mazel, 1983

2. Kaplan RF, Meyer RE, Stroebel CF: Alcohol dependence and responsivity to an ethanol stimulus as predictors of alcohol consumption. Br J Addict 78:259-267, 1983

3. Kaplan RF, Cooney NL, Baker LH, Gillespie RA, Meyer RE, Pomerleau OF: Reactivity to alcohol-related cues: physiological and subjective responses in alcoholics and nonproblem drinkers. J Stud Alcohol 46:267-272, 1985.

4. Pomerleau OF, Fertig J, Baker L, Cooney N.: Reactivity to alcohol cues in alcoholics and nonalcoholics: implications for a stimulus control analysis of drinking. Addict Behav 8:1-10, 1983

5. Cooney NL, Baker LH, Pomerleau OF, Josephy B: Salivation to drinking cues in alcohol abusers: toward the validation of a physiological measure of craving. Addict Behav 9:91-94, 1984

6. Kulkosky PJ: Brain-gut neuropeptides and the limitation of ethanol consumption. Neurosci Behav Rev 9:179-190, 1985

7. Rodin J: Has the distinction between internal versus external control of feeding outlived its usefulness? in Bray G (ed): Recent Advances in Obesity Research: II. London, Newman, 1978

8. Rodin J: Insulin levels, hunger and food intake: an example of feedback loops in body weight regulation. Health Psychol 4:1-15, 1985

9. Zito KA, Vickers G, Telford L, Roberts DC: Experimentally induced glucose intolerance increases oral ethanol intake in rats. Alcohol 1:257-261, 1984

10. Forsander O, Kohonen J, Suomalainen H: Physiological alcohol consumption. Q J Stud Alcohol 19:379-387, 1958

11. Goas JA, Pelham RW, Lippa AS: Endocrine factors contributing to the ethanol preference of rodents. Pharmacol Biochem Behav 10:557560,1979

12. Yung L, Gordis E, Hold J: Dietary choices and likelihood of abstinence among alcoholic patients in an outpatient clinic. Drug Alcohol Depend 12:355-362, 1983

13. Morgan MY: Alcohol and the endocrine system. Br Med Bull $38: 35-42,1982$

14. Cerasi E, Efendic S, Luft R: Dose-response relation between plasma-insulin and blood-glucose levels during oral glucose loads in prediabetic and diabetic subjects. Lancet 1:794-797, 1973

15. Margraf HW, Moyer CA, Ashford LE, Lavalle LW: Adrenocortical function in alcoholics. J Surg Res 7:55-62, 1967

16. Mendelson JH, Ogata M, Mello NK, Adrenal function and alcoholism, 1. Serum cortisol. Psychosom Med 33:145-157, 1971 